\title{
DES COUPLES «INTERPRÉTANTS » PLUTÔT QUE DES REPRÉSENTATIONS : LA DÉMARCHE DE BENVENISTE
}

\author{
Irène Fenoglio \\ ITEM (CNRS-ENS), Paris, France
}

\section{Résumé}

Dans la perspective d'Émile Benveniste, il sera difficile de s'en tenir aux termes de «représentation» et "opération». Plus pertinente est la notion d'interprétance, qui, empruntée à Pierce, prend dans la théorie benvenistienne du langage une valeur à la fois fondatrice (de la fonction du langage dans la société, de l'instance énonciative dans les activités de langage) et épistémologique. Elle sert de pivot entre la langue saussurienne et le discours, elle soutient la dimension méta de toute analyse linguistique. Cette notion d'interprétance se décline, chez Benveniste en divers dispositifs conceptuels couplés : désigner/signifier, sémiotique/sémantique, personne/non personne, selon les instances de langage visitées.

\section{Mots-clés}

Benveniste, interprétance, désignation/ signification, sémiotique/sémantique, écriture

\begin{abstract}
It's hard to comply with the terms of "representation" and "operation" in Emile Benveniste's view; far more relevant is the notion of interpretance, borrowed from interpretant's Peirce. In Benveniste's theory of language, the notion of interpretance is given a value that is both foundational (i.e. founding the function of language in society, of the enunciative instance of the acts of speaking) and epistemological. It acts as a pivot between saussurean langue on one hand and speech (discours) on the other, supporting the meta dimension of any linguistic analysis. According to the different instances of language, Benveniste's notion of interpretance is at play through various double conceptual devices: to refer to/to signify (désigner/signifier), semiotic/semantic (sémiotique/sémantique), grammatical person/non-person.
\end{abstract}

\section{Keywords}

Benveniste, interpretance, semiotics/ semantic, reference/signification, writing

\section{RELATION D'INTERPRÉTANCE}

Benveniste hérite de la conception saussurienne de la langue. Dans la note suivante $^{1}$, préparatoire à son cours de 1963-1964, il l'explicite clairement: 


\begin{tabular}{|c|c|}
\hline 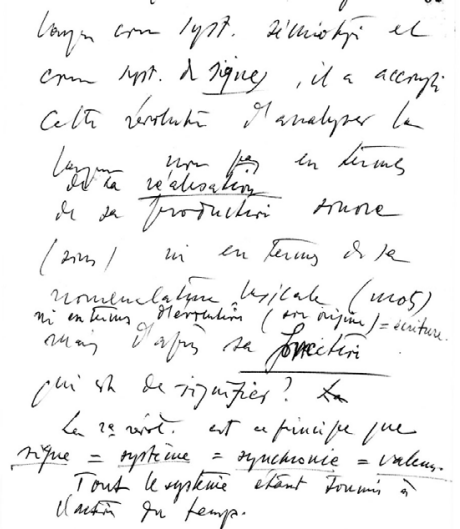 & 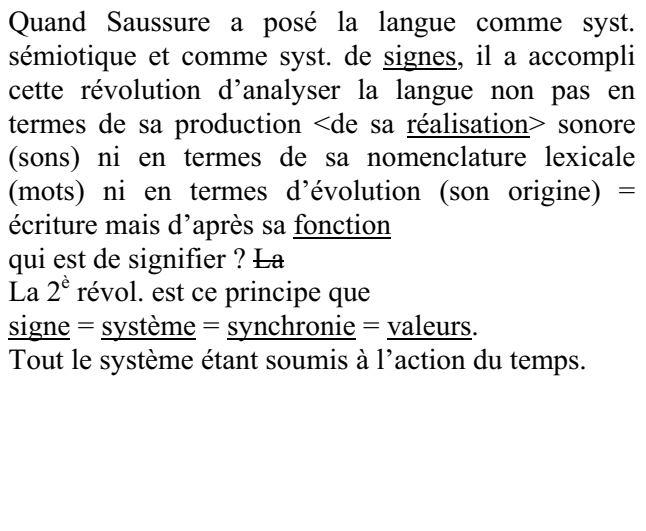 \\
\hline
\end{tabular}

Et dans celle-ci ${ }^{2}$ il place la langue à l'intérieur de l'ensemble des Sciences humaines :

\begin{tabular}{|c|c|}
\hline 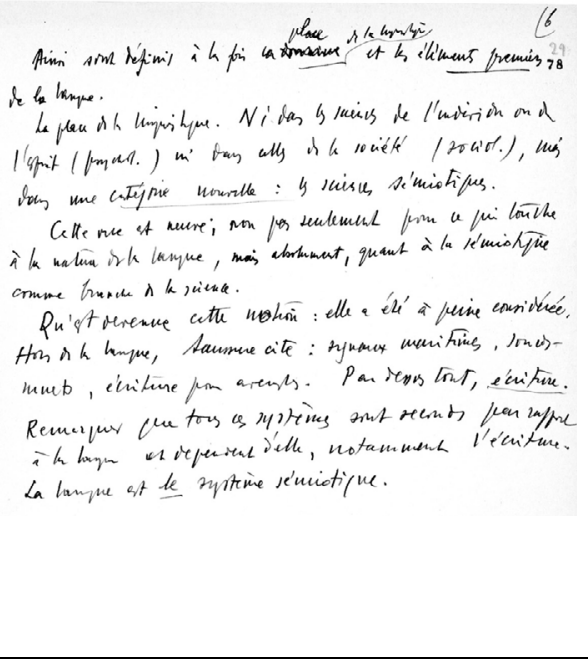 & $\begin{array}{l}\text { Ainsi sont définis à la fois le/a demaine place } \\
\text { <de la linguistique }>\text { et les éléments premiers } \\
\text { de la langue. } \\
\text { La place de la linguistique. Ni dans les } \\
\text { sciences de l'individu ou de l'esprit } \\
\text { (psychol.) ni dans celles de la société } \\
\text { (sociol.), mais dans une catégorie nouvelle : } \\
\text { les sciences sémiotiques. } \\
\text { Cette vue est neuve; non pas seulement pour } \\
\text { ce qui touche à la nature de la langue, mais } \\
\text { absolument, quant à la sémiotique comme } \\
\text { branche de la science. } \\
\text { Qu'est devenue cette notion: elle a été à peine } \\
\text { considérée. Hors de la langue, Saussure cite : } \\
\text { signaux maritimes, [ill.], écriture pour } \\
\text { aveugles. Par dessus tout, écriture. Remarquer } \\
\text { que tous ces systèmes sont seconds par rapport } \\
\text { à la langue et dépendent d'elle, notamment } \\
\text { l'écriture. La langue est le système } \\
\text { sémiotique. }\end{array}$ \\
\hline
\end{tabular}

Se soutenant de Saussure et, sans s'en départir, il développe cette question d'espace des sciences par des avancées à la fois critiques et complémentaires. Tout en partant fermement du socle saussurien, il s'en détache en opérant un renversement d'épistémé. Pour Saussure la langue est un système de signes très particulier certes, mais demeurant parmi d'autres systèmes de signes à l'intérieur d'une sémiologie englobante : 
[pour Saussure] la linguistique fait partie d'une science qui n'existe pas encore, qui s'occupera des autres systèmes du même ordre dans l'ensemble des faits humains, la SÉMIOLOGIE. (Benveniste 1980c [1969], p. 47)

Pour Benveniste, la langue est le système de signes qui englobe l'ensemble de tous les autres systèmes de signes. C'est à l'intérieur de cette réflexion que Benveniste est amené à utiliser la notion d'interprétance, venue de Peirce, chez qui elle est un élément pivot de sa/ses définition(s) du signe. Pour Peirce l'interprétant est un médiateur qui devient lui-même le signe d'un autre interprétant, engageant ainsi une semiosis illimitée. Benveniste utilise ce type de relation, à l'intérieur de l'exercice du langage. Il consacre d'ailleurs toute la première partie de son article «Sémiologie de la langue » à Peirce et à Saussure, posant leur conception au départ de sa réflexion pour mieux s'en détacher. Il discute les conceptions de Peirce avec un certain nombre de détails.

Poursuivie à travers une vie entière, la réflexion [de Peirce] s'est donné un appareil de plus en plus complexe de définitions visant à répartir la totalité du réel, du conçu et du vécu dans les différents ordres de signes. Pour construire cette «algèbre universelle des relations », Peirce a posé une triple division des signes, en ICONES, INDEX et SYMBOLES, [...]

En ce qui concerne la langue, Peirce ne formule rien de précis ni de spécifique. Pour lui la langue est partout et nulle part. Il ne s'est jamais intéressé au fonctionnement de la langue, si même il y a prêté attention. [...] La langue se réduit pour lui aux mots et ceux-ci sont bien des signes mais ils ne relèvent pas d'une catégorie distincte ou même d'une espèce constante. (ibid., p. 44)

En revanche, souligne Benveniste, «pour Saussure, à la différence de Peirce, le signe est d'abord une notion linguistique, qui, plus largement, s'étend à certains ordres de faits humains et sociaux.» (ibid., p. 48)

L'interprétance n'est pas une représentation ni même une relation d'interpréta$t_{i o n}{ }^{3}$. L'interprétance renvoie à une dynamique, un processus qui désigne la capacité d'un système sémiotique à transposer, transcrire un autre système ou à renvoyer à lui.

Benveniste pose trois types de relations entre systèmes sémiotiques : une relation d'engendrement

Cette RELATION D'ENGENDREMENT vaut entre deux système distincts et contemporains, mais de même nature, dont le second est construit à partir du premier et remplit une fonction spécifique

L'écriture ordinaire engendre l'écriture sténographique; l'alphabet normal engendre l'alphabet Braille.

3 Après Benveniste, en 1988, Umberto Eco reprend la notion, dans la perspective peircienne, pour préciser qu'à la différence de l'interprétation, elle n'est pas le fait d'un individu social inscrit dans une histoire (contresens de certains commentateurs de Peirce) et qu'elle est une opération formelle qui implique «à la fois une norme sociale ou habitus collectif déjà là et la détermination ici et maintenant d'un esprit qui intériorise cette norme» (Eco 1988, p. 108). 
une relation d'homologie

la RELATION D'HOMOLOGIE établit une corrélation entre les parties de deux systèmes sémiotiques.

l'homologie que Panofsky voit entre l'architecture gothique et la pensée scholastique

une relation d'interprétance

Nous désignons ainsi celle que nous instituons entre un système interprétant et un système interprété. [...] c'est le rapport entre les systèmes qui articulent, parce qu'ils manifestent leur propre sémiotique, et systèmes qui sont articulés et dont la sémiotique n'apparaît qu'à travers la grille d'un autre mode d'expression. (ibid., p. 60-61)

\section{LA LANGUE DÉSIGNE ET SIGNIFIE PLUTÔT QU'ELLE NE «REPRÉSENTE»}

Benveniste n'est pas un philosophe, il n'est pas un linguiste purement spéculatif. Il cherche, essaie et invente mais dans une combinaison toujours mesurée d'empirie et de réflexions théoriques. Ses réflexions ne sont jamais hors-sol. C'est dans cet ancrage empirique que l'on peut considérer son élaboration sur le rapport désignersignifier qu'il appuie sur la conception saussurienne de la langue. Empirie ne signifie pas recherche d'une substantialité.

L'apport de Saussure consiste en ceci: «Le langage, dit-il, est forme, non substance. » Il n'y a absolument rien de substantiel dans le langage. Toutes les sciences de la nature trouvent leur objet tout constitué. La linguistique, elle, et c'est ce qui la différencie de toute autre discipline scientifique, s'occupe de quelque chose qui n'est pas objet, pas substance, mais qui est forme. S'il n'y a rien de substantiel dans le langage, qu'y a-t-il? Les données du langage n'existent que par leurs différences, elles ne valent que par leurs oppositions. On peut contempler un caillou en soi, tout en le rangeant dans la série des minéraux. Tandis qu'un mot, à lui seul, ne signifie absolument rien. Il n'est que par opposition, par «vicinité » ou par différenciation avec un autre, un son par rapport à un autre son, et ainsi de suite. (Benveniste 1980a [1968], p. 31. C'est moi qui souligne.)

Et Benveniste va plus loin dans la mesure où il n'imagine pas la visée d'un objet linguistique sans méthodologie appropriée.

Le grand changement survenu en linguistique tient précisément en ceci : on a reconnu que le langage devait être décrit comme une structure formelle, mais que cette description exigeait au préalable l'établissement de procédures et de critères adéquats, et qu'en somme la réalité de l'objet n'était pas séparable de la méthode propre à le définir. (Benveniste 1976c [1962], p. 119. C'est moi qui souligne.) 
«La langue signifie», toutes les analyses et propositions partent de cette constatation que Benveniste emprunte à Saussure et qu'il va développer en « dépassant » Saussure. Dans sa toute dernière leçon de linguistique générale, celle du $1^{\text {er }}$ décembre 1969, il revient au problème de la signification: "La langue entière est informée et articulée par la signification.» (2012, p. 141); «On ne peut étudier le sens hors de la langue, ni la langue hors du sens» (ibidem, p. 142).

Durant toute son expérience linguistique, Benveniste ne cesse de s'interroger sur le rapport forme/sens; en 1962, dans son article «Les niveaux de l'analyse linguistique », il insiste sur la nécessité de ce questionnement:

Voici que surgit le problème qui hante toute la linguistique moderne, le rapport forme: sens que maints linguistes voudraient réduire à la seule notion de la forme, mais sans parvenir à se délivrer de son corrélat, le sens. Que n'a-t-on tenté pour éviter, ignorer, ou expulser le sens ? On aura beau faire : cette tête de méduse est toujours là, au centre de la langue, fascinant ceux qui la contemplent. (Benveniste 1976c [1962], p. 126. C'est moi qui souligne.)

La langue désigne et signifie : deux opérations inévitables et inséparables. Dans son Avant-propos au Vocabulaire des institutions indo-européennes, Benveniste qualifie cette distinction d' " essentielle », «à défaut de laquelle tant de discussion sur le sens sombrent dans la confusion» (1969b, p. 12) et la quatrième de couverture du volume 2 précise :

Partant des correspondances entre les formes historiques, on cherche, au-delà des désignations, qui sont souvent très divergentes, à atteindre le niveau profond des significations qui les fondent, pour retrouver la notion première de l'institution comme structure latente, enfouie dans la préhistoire linguistique

En faisant appel à ce couple, Benveniste opère une distinction théorique :

C'est en vertu d'un procès distinct, tout pragmatique et secondaire que *peku dont le sens était «possession mobilière» a été appliqué spécifiquement à la réalité dite «bétail». Il faut distinguer dans cette analyse les deux plans théoriques : celui de la signification et celui de la désignation (ibid., p. 53)

Dans les Dernières leçons (leçon 14) Benveniste explique qu'il faut analyser ce « procès linguistique » de dénomination qui fait que, si nous « savons déjà » ce que désignent les termes employés, il est très important de savoir ce qu'ils signifient. Les termes désignent un référent mais que signifient-ils hors de cette désignation? L'important est donc pour Benveniste de «distinguer entre la désignation et la signification ».

«Comment une langue dénomme l'acte qui lui donne expression écrite », c'est la question que Benveniste pose au début de sa leçon 14 ; l'analyse s'attache à la façon dont se désignent les actes de lire et d'écrire : la signification dépasse la désignation du geste. 
Chez Homère, /grapho/ ne signifie que «gratter», «érafler», «entailler la chair », par exemple (Il., XVII, 599). Postérieurement, « entailler la pierre pour inscrire une trace». [...]

En latin, de même : /scribo/ signifie «érafler», «gratter».

En allemand récent, /schreiben/, mais en gotique,/meljan/ (voir l'allemand, /mahlen/, « peindre ») : « noircir», « salir »; grec, /melas/, « salir de couleur »). Il s'agit donc de traces peintes. Ce n'est plus de la gravure, mais de la peinture. En norrois, /rita/, en vieil anglais, /writan/: sens : «tailler».

En slave, emprunt à l'iranien /pisati/, au sens d'«écrire».

En vieux perse, /dipi-/ est le terme dénommant l'« inscription». Et celui pour «écrire » est tout à fait indépendant. Il est composé d'un préverbe /ni-/ et d'un radical /pis-/. / Ni-/ indique un procès réalisé par « descente» : « inscrire» et/pis-/, le procès «peindre», "piquer» (voir la technique du tatouage). Le radical a été emprunté par le vieux slave et le verbe est apparenté étymologiquement au latin /pingo/, « dessiner », « peindre ».

De même, les éléments de l'écriture, les lettres, sont à interroger : en grec, /gramma/ est dérivé de /grapho/, mais /litera/ est d'origine encore inconnue. (Benveniste 2012, p. 124)

Cette «analyse de terminologie» (ibid., p. 121) met en jeu les deux opérations désigner et signifier; elles sont couplées: distinctes et nécessaires mais indissociables. Certes les signes d'un système de langue désignent un objet, un acte, le produit d'une opération, mais comprendre profondément l'évolution d'une langue et la façon dont elle fonctionne nécessite d'expliciter ce que le signe signifie, ici et maintenant et dans son histoire. Dans le chapitre 8 du Vocabulaire, sur «la fidélité personnelle», Benveniste remarque :

On constate ici à plein la différence entre la signification et la désignation et la distance qui peut les séparer, au point que souvent la désignation ne laisse en rien présumer de la signification si l'on ne dispose pas de repères lexicaux. (1969b, p. 108)

\section{SÉmiotique ET SÉMANTiQue EN DISCOURS}

Mais lorsque nous opérons par désigner-signifier nous restons en langue. On sait que Benveniste établit qu'une autre instance de fonctionnement du langage doit être mise au jour : l'instance du discours, espace de la phrase, de l'énonciation. La langue signifie alors à deux niveaux : le sémiotique et le sémantique, couplés eux aussi, distinguables mais intrinsèquement liés.

En discours on ne peut s'abstraire de la langue. C'est ainsi que le sémiotique supporte la langue, c'est-à-dire l'ensemble du phénomène couplé: désignersignifier. L'énonciation est la situation qui permet d'évaluer, de constater la signification.

Benveniste produit de nombreuses définitions du couple sémiotique/sémantique. Je citerai celle de son entretien de 1968 avec Pierre Daix dans lequel il précise que 
« si on ne commence pas par reconnaître cette distinction, je crains qu'on reste dans le vague.» (1980b [1968], p. 22):

Je pose qu'il y a deux domaines ou deux modalités de sens, que je distingue respectivement comme sémiotique et sémantique. Le signe saussurien est en réalité l'unité sémiotique, c'est-à-dire l'unité pourvue de sens. Est reconnu ce qui a un sens; tous les mots qui se trouvent dans un texte français, pour qui possède cette langue ont un sens [...] Le niveau sémiotique, c'est ça: être reconnu comme ayant ou non un sens. Ça se définit par oui, non.

Le sémantique, c'est le « sens » résultant de l'enchaînement, de l'appropriation à la circonstance et de l'adaptation des différents signes entre eux. Ça c'est absolument imprévisible. C'est l'ouverture vers le monde. Tandis que le sémiotique, c'est le sens refermé sur lui-même et contenu en quelque sorte en lui-même. (ibid., p. 21. C'est moi qui souligne.)

C'est dans cet espace de l'imprévisible, exprimé pourtant par des contraintes de langue, que se constitue l'énonciation.

Et pour mettre en œuvre ces dispositifs épistémologiques qui ont une grande puissance d'intelligibilité du phénomène langagier, Benveniste a repéré des outils analytiques que l'on connaît et qu'il englobe dans ce qu'il appelle «l'appareil formel de l'énonciation »

Il faudrait entièrement citer ce magnifique article que demeure «De la subjectivité dans le langage » paru en 1958 dans Journal de psychologie, tant il est dense, riche, innovant à un point extrême.

C'est dans et par le langage que l'homme se constitue comme sujet; parce que le langage seul fonde en réalité, dans sa réalité qui est celle de l'être, le concept d'« ego».

La «subjectivité» dont nous traitons ici est la capacité du locuteur à se poser comme «sujet». Elle se définit, non par le sentiment que chacun éprouve d'être lui-même (ce sentiment, dans la mesure où on peut en faire état, n'est qu'un reflet), mais comme l'unité psychique qui transcende la totalité des expériences vécues qu'elle assemble, et qui assure la permanence de la conscience. Or nous tenons que cette «subjectivité», qu'on la pose en phénoménologie ou en psychologie, comme on voudra, n'est que l'émergence dans l'être d'une propriété fondamentale du langage. Est « ego » qui dit « ego ». Nous trouvons là le fondement de la «subjectivité » qui se détermine par le statut linguistique de la «personne» (1976b [1958], p. 259-260. C'est moi qui souligne.)

On voit bien là qu'il n'y a pas représentation mais constitution de la subjectivité. Non seulement le langage est le seul moyen de l'exprimer mais, plus que cela, en réciprocité dirai-je,

Le langage n'est possible que parce que chaque locuteur se pose comme sujet, en renvoyant à lui-même comme je dans son discours. De ce fait, je pose une autre personne, celle qui, tout extérieure qu'elle est à «moi», devient mon écho auquel je dis $t u$ et qui me dit $t u$. [...] 
Ainsi tombent les vieilles antinomies du « moi » et de l' « autre», de l'individu et de la société. [...] C'est dans une réalité dialectique englobant les deux termes et les définissant par relation mutuelle qu'on découvre le fondement linguistique de la subjectivité. (ibid., p. 260. C'est moi qui souligne.)

Dès 1946, Benveniste élabore ce qui restera son essentiel apport à la linguistique générale :

Une théorie linguistique de la personne verbale ne peut se constituer que sur la base des oppositions qui différencient les personnes; et elle se résumera tout entière dans la structure de ces oppositions. Pour la déceler, on pourra partir des définitions que les grammairiens arabes emploient. Pour eux, la première personne est al-mutakallimu, "celui qui parle»; la deuxième al-muhatabu, «celui à qui on s'adresse»; mais la troisième est al-ya'ibu, «celui qui est absent». Dans ces dénominations se trouve impliquée une notion juste des rapports entre les personnes; juste surtout en ce qu'elle révèle la disparité entre la $3^{\text {éme }}$ personne et les deux premières. (1976a [1946], p. 227-228)

Il continue son raisonnement :

Il s'ensuit que, très généralement, la personne n'est propre qu'aux positions «je » et « tu ». La $3{ }^{\text {éme }}$ personne est, en vertu de sa structure même, la forme nonpersonnelle de la flexion verbale.

De fait elle sert toujours quand la personne n'est pas désignée et notamment dans l'expression dite impersonnelle. Nous retrouvons ici la question des impersonnels, vieux problème et débat stérile tant que l'on persiste à confondre «personne» et «sujet». (ibid., p. 230)

Il fait aboutir son analyse sur la mise en relief de « deux corrélations constantes » et très importantes dans la distinction qu'elles impliquent:

1. Corrélation de personnalité opposant les personnes je/tu à la non-personne il ;

2. corrélation de subjectivité, intérieure à la précédente et opposant je à tu. (ibid., p. 235)

Nous sommes donc bien encore dans des distinctions couplées, c'est-à-dire des systèmes de relations.

\section{L’ÉCRITURE NE «REPRÉSENTE » PAS LA LANGUE, ELLE LA CONSTITUE}

\section{LINGUISTIQUEMENT}

Le chapitre VI du Cours de Linguistique générale s'intitule « Représentation de la langue par l'écriture». Pour le Saussure du Cours de linguistique générale, la langue et l'écriture sont deux systèmes de signes distincts mais le second «représente» le premier: donc l'écriture représente la langue qui lui serait préalable. Face à cette vision Benveniste offre une autre perspective ; il critique le flou du concept de «représentation» utilisé par Saussure. 
L'écriture est la dernière question de Benveniste; il y reprend toutes les données de linguistique générale mais, montre-t-il et insiste-t-il, l'écriture ajoute quelque chose au fonctionnement du langage: l'incorporation de l'épistémologie intrinsèque à l'étude du langage. Il remonte à la source de l'écriture pour comprendre qu'elle est un système qui permet à la langue de s'autosémiotiser, de se constituer en se formalisant, en s'auto-formalisant. L'écriture n'est plus une application secondaire mais une création constituante de forme, d'ordre et de méta-communication. C'est en exposant sa longue réflexion sur l'écriture qu'il va spécifier la relation d' " autosémiotisation » que la langue entretient avec elle-même: l'écriture fait voir la langue ${ }^{4}$.

Voici comment Benveniste résumait cette partie du cours de 1968-1969 portant sur l'écriture et aujourd'hui publié dans les Dernières leçons:

Enfin, nous avons examiné les rapports entre la langue et le système sémiotique
constitué par l'écriture. Au terme d'un examen détaillé qui nous a fait parcourir
les différents modèles d'écriture attestés dans l'histoire, il nous est apparu que,
contrairement à l'idée admise partout, l'écriture ne constitue pas un système
distinct. C'est le prolongement ou la projection de la langue même, et donc la
même situation à l'égard des systèmes extra-linguistiques. Nous voyons dans
l'écriture l'instrument et la manifestation du procès d'auto-sémiotisation de
la langue. (1969a, p. 364 . C'est moi qui souligne.)

L'avancée de Benveniste sur cette question de l'écriture est clairement exprimée au début de la leçon 12 :

On dirait que l'écriture a été et qu'elle est en principe un moyen parallèle à la parole de raconter les choses ou de les dire à distance et que progressivement l'écriture s'est littéralisée en se conformant à une image de plus en plus formelle de la langue. (2012, p. 114)

Les termes employés par Benveniste sont importants : moyen parallèle, l'écriture n'a pas toujours été le reflet de la parole. Elle s'est «littéralisée», le mot est parfaitement choisi, il faut le prendre en tous les sens qu'il ouvre: passage de l'icône à la lettre, et passage de l'utilisation linguistique à la représentation $d u$ linguistique, autrement dit de la langue.

L'écriture rend visible ces mouvements; elle passe de sa «fonction instrumentale» (instrument de sémiotisation) à une «fonction représentative»:

d'instrument à iconiser le réel, c'est-à-dire le référent, à partir d'un discours, elle devient peu à peu le moyen de représenter le discours lui-même, puis les éléments $\mathrm{du}$ discours, puis les éléments de ces éléments (sons/lettres). (ibid., p. 115)

Benveniste insiste sur le fait que, avec l'écriture, le locuteur « doit prendre conscience de la langue comme réalité distincte de l'usage qu'il en fait» (ibid., p. 93). 
Cela veut dire que le parlant s'arrête sur la langue au lieu de s'arrêter sur les choses énoncées ; il prend en considération la langue et la découvre signifiante ; il remarque des récurrences, des identités, des différences partielles et ces observations se fixent dans des représentations graphiques qui objectivent la langue et qui suscitent en tant qu'images la matérialité même de la langue. L'écriture et tout particulièrement l'écriture alphabétique est l'instrument de l'auto-sémiotisation de la langue. Comment? En vertu des propositions suivantes : 1) La langue est le seul système signifiant qui puisse se décrire luimême dans ses propres termes. La propriété métalinguistique est bien propre à la langue du fait qu'elle est l'interprétant des autres systèmes. 2) Mais pour que la langue se sémiotise, elle doit procéder à une objectivation de sa propre substance. L'écriture devient progressivement l'instrument de cette objectivation formelle. (ibid., p. 113. C'est moi qui souligne.)

Il s'agit de la proposition essentielle de Benveniste : la langue se sémiotise ellemême et ne peut être sémiotisée que par elle-même et ce processus s'effectue au moyen de l'écriture; on est loin de la simple représentation:

on n'aurait pas pu réfléchir sur l'analyse du langage parlé si l'on n'avait pas disposé de ce «langage visible» qu'est l'écriture.

Ainsi l'écriture qui a permis la mise au jour de la langue, participe de l'interprétance par la langue des autres systèmes de signes. Derrida serait d'accord, lui qui écrivait : «Il n’y a pas de signe linguistique avant l'écriture » (1967, p. 25).

\section{Conclusion: UnE ChaÎNE D’INTERPRÉTANCE}

La langue interprète le monde et la société grâce à sa double dimension désignersignifier. Dans «Sémiologie de la langue » en passant en revue plusieurs systèmes dont «les systèmes où la signifiance est imprimée par l'auteur à l'œuvre» (par exemple la signifiance de l'art) et les systèmes où la signifiance est « inhérente aux signes eux-mêmes» (1980c [1969], p. 59), Benveniste clarifie le fait que «la signifiance de la langue est la signifiance même, fondant la possibilité de tout échange et de toute communication, par là de toute culture.» (ibid., p. 60).

Toute sémiologie d'un système non-linguistique doit emprunter le truchement de la langue, ne peut donc exister que par et dans la sémiologie de la langue [...]. La langue est l'interprétant de tous les autres systèmes, linguistiques et non-linguistiques. (ibid., p. 60)

La langue est l'interprétant de tous les systèmes sémiotiques. Aucun autre système ne dispose d'une "langue» dans laquelle il puisse se catégoriser et s'interpréter selon les distinctions sémiotiques, tandis que la langue, peut, en principe, tout catégoriser et interpréter, y compris elle-même. (ibid., p. 61-62. C'est moi qui souligne.)

L'affirmation - répétée - est sans ambiguïté. C'est à ce lieu-là de réflexion et d'insistance que Benveniste opère un renversement épistémique par rapport à Saussure. Benveniste prend la peine d'expliciter et surtout de montrer que: 
La langue nous donne le seul modèle d'un système qui soit sémiotique à la fois dans sa structure formelle et dans son fonctionnement:

$1^{\circ}$ elle se manifeste par l'énonciation, qui porte référence à une situation donnée ; parler, c'est toujours parler de ;

$2^{\circ}$ elle consiste formellement en unités distinctes, dont chacune est un signe ; $3^{\circ}$ elle est produite et reçue dans les mêmes valeurs de référence chez tous les membres d'une communauté ;

$4^{\circ}$ elle est la seule actualisation de la communication intersubjective.

Pour toutes ces raisons, la langue est l'organisation sémiotique par excellence. Elle donne l'idée de ce qu'est une fonction de signe, et elle est seule à en offrir la formule exemplaire. De là vient qu'elle peut seule conférer - et elle confère effectivement-à d'autres ensembles la qualité de systèmes signifiants en les informant de la relation de signe. [...] La nature de la langue, sa fonction représentative, son pouvoir dynamique, son rôle dans la vie de relation font d'elle la grande matrice sémiotique, la structure modélisante dont les autres structures reproduisent les traits et les modes d'action.

À quoi tient cette propriété ? [...] Nous le découvrirons en prenant conscience de ce fait que la langue signifie d'une manière spécifique et qui n'est qu'à elle, d'une manière qu'aucun autre système ne reproduit. Elle est investie d'une DOUBLE SIGNIFIANCE. C'est là proprement un modèle sans analogue. La langue combine deux modes distincts de signifiance que nous appelons le mode SÉMIOTIQUE et le mode SÉMANTIQUE de l'autre. (ibid., p. 62-63. C'est moi qui souligne.)

L'énonciation interprète la parole (orale ou écrite) par la double instance du discours: sémiotique-sémantique et par la constitution d'un sujet qui parle. L'écriture interprète la langue et permet l'élaboration linguistique. Si l'on peut parler d'opérations chez Benveniste il reste difficile de rendre compte de sa théorie du langage en termes de représentation.

Dans «Catégories de pensée et catégorie de langue», tout en montrant le lien entre la représentation du monde et les langues particulières qui, chaque fois, l'expriment par la double opération de désigner-signifier, il prend la peine de préciser :

Aucun type de langue ne peut par lui-même et à lui seul ni favoriser ni empêcher l'activité de l'esprit. L'essor de la pensée est lié bien plus étroitement aux capacités des hommes, aux conditions générales de la culture, à l'organisation de la société qu'à la nature particulière de la langue. Mais la possibilité de la pensée est liée à la faculté de langage, car la langue est une structure informée de signification, et penser, c'est manier les signes de la langue. (1976b [1958], p. 74. C'est moi qui souligne.)

Ainsi, si «nous ne saisissons la pensée que déjà appropriée aux cadres de la langue $»$, c'est que

la forme linguistique est non seulement la condition de transmissibilité, mais d'abord la condition de réalisation de la pensée. (ibid., p. 64. C'est moi qui souligne.) 
Ce système de relations couplées interprétantes permet à Benveniste d'éviter les dichotomies et en particulier la dichotomie la plus nocive pour la compréhension du langage: celle de la pensée et du langage. Cette vision du langage pris dans l'expérience humaine, elle-même prise dans la nature, est étrangère à toute la linguistique qui s'est développée depuis Chomsky et tous ses dérivés qui considèrent que le langage n'est qu'un instrument pour la pensée existant préalablement et pour lesquels la modélisation a priori des formes langagières parfois séparées de leur «signification» vaut connaissance du fonctionnement réel.

Je rappelle pour finir un passage de l'Avant-propos du Vocabulaire des institutions indo-européennes où il explicite la notion d'indo-européen, une « famille de langue», l'indo-européen :

Le miracle alors que les phases de ces migrations et implantations nous restent inconnues, est que nous puissions désigner à coup sûr les peuples qui ont fait partie de la communauté initiale [...]. La raison en est la langue et seulement la langue. La notion d'indo-européen vaut d'abord comme notion linguistique et si nous pouvons l'élargir à d'autres aspects de la culture, ce sera encore à partir de la langue (1969b, p. 7-8).

\section{BIBLIOGRAPHIE}

\section{Sources primaires}

Annuaire du Collège de France, $69^{\text {ème }}$ année, 1969a. Paris, Imp. Nationale.

Fonds Émile Benveniste de la Bibliothèque nationale de France.

Benveniste, Émile, 1969b. Vocabulaires des institutions indo-européennes, Paris, Les Éditions de Minuit.

- 1976a [1946]. «Structure des relations de personne dans le verbe», Problèmes de linguistique générale, 1, Paris, Gallimard, 225-236.

- 1976b [1958]. "Catégories de pensée et catégories de langue», Problèmes de linguistique générale, 1, Paris, Gallimard, 63-74.

— 1976c [1958]. «De la subjectivité dans le langage », Problèmes de linguistique générale, 1, Paris, Gallimard, 258-266.

- 1976d [1962]. «Les niveaux de l'analyse linguistique», Problèmes de linguistique générale, 1, Paris, Gallimard, 119-131.

— 1980a [1968]. «Ce langage qui fait l’histoire», Problèmes de linguistique générale, 2, Paris, Gallimard, 29-40.

— 1980b [1968]. «Structuralisme et linguistique», Problèmes de linguistique générale, 2, Paris, Gallimard,11-40.

— 1980c [1969], «Sémiologie de la langue », Problèmes de linguistique générale, 2, Paris, Gallimard, 43-66.

- 2012. Dernières leçons, texte établi et introduit par Jean-Claude Coquet et Irène Fenoglio, EHESS-Gallimard-Seuil. 


\section{Sources secondaires}

Derrida, Jacques, 1967. De la grammatologie, Paris, Les Éditions de Minuit. Eco, Umberto, 1988. Sémiotique et philosophie du langage, Paris, PUF.

Fenoglio, Irène, 2016. "L'écriture au fondement d'une civilisation laïque », Fenoglio, Irène, Coquet Jean-Claude, Kristeva Julia, Malamoud, Charles, et Quignard, Pascal, Autour d'Emile Benveniste. Sur l'écriture, Paris, Seuil, 153-236. 